\title{
Uncertainty and the Cost of Reversal
}

GIOVANNI IMMORDINO

giimmo@tin.it

CSEF, Università di Salerno; Giovanni Immordino, Dipartimento di Scienze Economiche Università di Salerno, Via Ponte Don Melillo 80084, Fisciano (SA), Italy

Received February 26, 2002; Revised March 09, 2005

\begin{abstract}
For standard irreversibility theory the prospect of acquiring better information in the future should induce more flexible decisions: the "irreversibility effect". This result relies on the definition of an irreversible position as one that would be technically or economically impossible to reverse. In practice, many positions can be reversed at an affordable cost. In this case an increase in informativeness alone is not enough to bias decisions in favour of more flexibility. We look for restrictions on decision sets, information structures and preferences that make possible to study the effect of information on flexibility.
\end{abstract}

Key words: irreversibility, information structures

JEL Classification No.: D81, D9.

\section{Introduction}

When a new product or a cost saving production process is invented, policymakers have to decide whether to allow it or not. In taking this decision they try to evaluate expected costs and benefits, but the possible effects of innovations on the environment or on consumers' health are generally imperfectly known. Consider for example the storage of nuclear wastes. A possibility currently under study is to store them in a geological storage 500 to 1000 meters deep. This technology is characterized by high irreversibility (in the event of a leakage of radioactivity in the environment, it is very costly to recoup the wastes).

In this context imperfect scientific knowledge is an important source of uncertainty. There are ongoing studies about how to reduce the radioactivity of wastes (a technique known as "separation and transmutation" ). If these studies succeed in discovering a new process for treating and recycling wastes, decision makers would prefer ex-post to recoup the wastes and treat them with the new technique.

These considerations raise the following question: which storing technology should we adopt today if we expect more progress in scientific research?

In this as in many other examples, the value of information comes from the policymaker ability to adapt decisions to information. Flexibility makes more information valuable. If flexibility comes at no cost, the decision maker will always opt for maximum flexibility. But flexibility is costly. In standard irreversibility theory (Arrow and Fisher [1974]; Henry [1974]; Epstein [1980]; Freixas and Laffont [1984]; Jones and Ostroy [1984]) the decision 
maker chooses a level of today's decision variable. This choice results from an intertemporal trade off between raising today's utility and constraining tomorrow's choices. The main finding is that the prospect of acquiring better information in the future will induce the decision maker to bias is ex ante decision in favour of more flexibility, the "irreversibility effect".

This result relies on the definition of an irreversible position as one that would be technically or economically impossible to reverse. ${ }^{2}$

This notion of irreversibility is an extreme one. In practice, many positions can be reversed at an affordable cost. The purpose of this paper is to show that, in this often more realistic case, the irreversibility effect may not hold and to spell out the assumptions required for the effect to hold or not.

An increase in informativeness alone is not enough to bias decisions in favour of more flexibility. Our results can be summarized as follows. First, if the first period choice is restricted to be 'all or nothing' and if the first period choice is technically or economically irreversible then better information promotes flexibility, as in Epstein [1980]. But a lower cost of reversal can make the rigid position preferable. Second, if we look at the special information improvements that live posterior distributions unchanged, we find that the value of remaining flexible tends to decrease with better information. Finally, if relative risk aversion is constant (CRRA) and lower than 1, the irreversibility effect holds even when a position is not economically or technically irreversible.

The paper is organized as follows. In Section 2 we present the model and we clarify the role of risk and uncertainty. In Section 3 we study the comparative statics of a better information structure: (i) when the set of actions available is restricted to be binary; (ii) when information improvements live posterior distributions unchanged; and (iii) when utility has constant relative risk aversion. Some concluding comments are provided in the last section.

\section{The model}

Standard irreversibility theory considers a model with two dates $i=1,2$; with a decision maker that maximizes the sum of the expected utility of the generations living at dates 1 and 2. Let $a$ in $\Re$ and $c$ in $\Re$ be the decision variables in period 1 and in period 2.c must belong to the choice set of the second period $\Psi(a)$, which may depend on the decision $a$ taken in the first period. $a$ indicates the degree of flexibility in a decision, increasing $a$ enlarges the opportunity set in the future. To keep things simple, $\Psi(a)$ is assumed to be the set of all $c$ that are less than $a: \Psi(a)=\{c \mid c \leq a\}$. The complementary set $\Psi^{\prime}(a)=\{c \mid c>a\}$ is the set of positions which are not attainable. In other words, the choice of $a$ is a completely irreversible decision. Either for technical or for economic reasons, this choice completely fixes the actions that will be available tomorrow. The objective function is assumed to be additively separable between the two periods and is written: $u(a)+v(c \tilde{x})$, where $\tilde{x}$ represents the randomness of the problem and utility functions $u$ and $v$ are assumed to be twice differentiable and strictly concave. Assume $\tilde{x}$ discrete with $n$ atoms $\left(x_{1}, x_{2}, \ldots, x_{n}\right)$. The decision maker has some prior beliefs about the distribution of $\tilde{x}$. At $t=1$ it is known that some information $\tilde{y}$ on the distribution of $\tilde{x}$ will be available before $t=2 .{ }^{3}$ This information will allow the decision maker to revise the distribution of $\tilde{x}$ according to 
Bayes' rule. The expected change in beliefs in turn has an impact on the initial choice of $a$. The standard irreversibility decision problem is thus the following:

$$
\max _{a} u(a)+E_{\tilde{y}} \max _{c \leq a} E_{\tilde{x} / \tilde{y}} v(c \tilde{x}) .
$$

We amend the standard model by allowing for a less extreme notion of irreversibility. After a signal $y$ is observed and before the second period choice $c$, the decision maker may enlarge the opportunity set by some costly action. In practice, many positions can be reversed at an affordable cost: nuclear wastes can be recouped and treated, the stock of $\mathrm{CO}_{2}$ in the atmosphere can be abated by planting forests, investments can be liquidated at a cost, and so on. Denote

$$
C(a, b) \begin{cases}=C(b) & \text { if } b>a \\ =0 & \text { if } b \leq a,\end{cases}
$$

the cost necessary to enlarge period 2 opportunity set from $\Psi(a)=\{c \mid c \leq a\}$ to $\Psi(b)=$ $\{c \mid c \leq b\}$. Where $C(b)$ is strictly increasing and convex. The 'new' irreversibility decision problem is thus the following:

$$
\max _{a} u(a)+E_{\tilde{y}} \max _{b}\left[\max _{c \leq b} E_{\tilde{x} / \tilde{y}} v(c \tilde{x})-C(a, b)\right] .
$$

As before the decision maker faces a trade off between raising today's utility and constraining tomorrow's choices. But differently from (2.1), the decision $a$ taken in the first period is not completely irreversible. In this setting $a$ is an irreversible position if for any $b>a$ and for any signal $y$ we have the condition that $E_{\tilde{x} / \tilde{y}} v(b \tilde{x})<C(b)$. Note that when a position is irreversible, the optimal intermediate decision is to choose $b=a$, so that we are back to the standard model.

\section{Comparative statics on information structures}

We denote $\pi_{y}(x)$ the probability of $\tilde{x}=x$ conditional on the reception of message $y$, $\pi_{y}=\left(\pi_{y}\left(x_{1}\right), \pi_{y}\left(x_{2}\right), \ldots, \pi_{y}\left(x_{n}\right)\right)$, and $S=\left\{\pi_{y} \in R_{+}^{n} \mid \sum_{i=1}^{n} \pi_{y}\left(x_{i}\right)=1\right\}$ the set of potential conditional distributions on $\tilde{x}$. Let us also denote

$$
V\left(b, \pi_{y}\right)=\max _{c \leq b} E_{\tilde{x} / \tilde{y}} v(c \tilde{x}),
$$

the value function for the second period, and

$$
J\left(a, \pi_{y}\right)=\max _{b}\left[V\left(b, \pi_{y}\right)-C(a, b)\right],
$$

the value function for the intermediate problem. 
Following Blackwell [1951] and Marschak and Miyasawa [1968] we say that a signal $\tilde{y}$ is more informative than a signal $\tilde{y}^{\prime}$ if and only if:

for any $\rho$ convex on $\mathrm{S}: E_{\tilde{y}} \rho\left(\pi_{y}\right) \geq E_{\tilde{y}^{\prime}} \rho\left(\pi_{y^{\prime}}\right)$.

Our main purpose is to determine the comparative statics of a better information structure. In our model, this means determining how a signal $\tilde{y}$ more informative than signal $\tilde{y}^{\prime}$ affects the efficient level $a$. From now on when we refer to two economies satisfying condition (3.3), we will talk of "a better information structure." As shown by Marschak and Miyasawa [1968], condition (3.3) is equivalent to the fact that all expected utility maximizers observing $\tilde{y}$ are at least as well off as when observing $\tilde{y}^{\prime}$. That is to say:

$$
E_{\tilde{y}} J\left(a, \pi_{y}\right) \geq E_{\tilde{y}^{\prime}} J\left(a, \pi_{y^{\prime}}\right)
$$

We thus have two equivalent definitions of a better information structure, (3.3) and (3.4). We can now rewrite problem (2.3) as:

$$
\max _{a} u(a)+E_{\tilde{y}} J\left(a, \pi_{y}\right) .
$$

We assume $J\left(a, \pi_{y}\right)$ to be concave and differentiable with respect to $a$. The next Lemma, which is a particular case of theorem 1 in Epstein [1980], provides a way to determine whether a better information structure increases or decreases flexibility, i.e., whether the irreversibility effect holds or not.

Lemma 1: A better information structure increases the degree of flexibility (increases a) if and only if $J_{a}\left(a, \pi_{y}\right)$ is convex in $\pi_{y}$.

Proof. Writing first order conditions both for $\tilde{y}$ and $\tilde{y}^{\prime}$ it is immediate that a better information structure increases $a$ if and only if $\left.E_{\tilde{y}} J_{a} a, \pi_{y}\right) \geq E_{\tilde{y}^{\prime}} J_{a}\left(a, \pi_{y^{\prime}}\right)$. Then definition (3.3) of better information structure gives the result.

More information in the future implies the optimal exposure to risk to be more sensitive to signals. More extreme signals push to switch decision more often in the second period. This yields an increase in risk ex ante. Remaining flexible is in effect like buying an option as to what later action will be taken. We would then expect that the better the information the greater the value of the option, that is, the greater the degree of flexibility. We show next that the intuition is true for problem (2.1), but it is not generally true for the more general problem (2.3).

Let us first review problem (2.1). It is easy to verify that the Kuhn-Tucker conditions for problem $J\left(a, \pi_{y}\right)=\max _{c \leq a} E_{\tilde{x} / \tilde{y}} v(c \tilde{x})$ imply that

$$
J_{a}\left(a, \pi_{y}\right)=\max \left\{0, E_{\tilde{x} / \tilde{y}} v^{\prime}(a \tilde{x}) \tilde{x}\right\} .
$$


The second function inside the maximum is linear and hence convex in the vector of probabilities. Since a maximum of two convex functions is itself convex, it follows that $J_{a}\left(a, \pi_{y}\right)$ is convex in $\pi_{y}$. Therefore, by the Lemma, the irreversibility effect holds.

We now move to problem (2.3). Use the second period value function (3.2) and the definition of the cost of reversal (2.2), to get:

$$
J_{a}\left(a, \pi_{y}\right) \begin{cases}=0 & \text { if } b^{*}\left(a, \pi_{y}\right) \leq a, \\ =C\left[b^{*}\left(a, \pi_{y}\right)\right] & \text { if } b^{*}\left(a, \pi_{y}\right)>a,\end{cases}
$$

or equivalently $J_{a}\left(a, \pi_{y}\right)=\max \left\{0, C\left[b^{*}\left(a, \pi_{y}\right)\right]\right\}$. Differently from before the second function inside the maximum is not linear in the vector of probabilities, given that $b^{*}$ is itself a function of $\pi_{y}$. Therefore, in general we are not able to say whether $J_{a}\left(a, \pi_{y}\right)$ is convex or concave in $\pi_{y}$ and to use the Lemma for signing the effect of a better information structure on the degree of flexibility. Next, we consider three cases where it is possible to study the effect of information on flexibility.

\subsection{The binary choice problem}

We consider a special case of our problem. Suppose for the moment that the decision maker is forced to make an all or nothing choice, i.e., $a$ can take only values zero or one. For example, the decision maker could be forced to store all nuclear wastes with one technology because of fixed costs. This binary first period choice applies most usefully to situations where the choice is between going ahead with what seems best at the moment versus waiting for more information $(a=1)$. The problem then becomes:

$$
\max _{a \in\{0,1\}} u(a)+E_{\tilde{y}} \max _{b \in\{0,1\}}\left[\max _{c \leq b} E_{\tilde{x} / \tilde{y}} v(c \tilde{x})-C(a, b)\right] .
$$

The standard result is the following. If the flexible choice $a=1$, is preferred in an economy with a given information $\left(i^{-}\right)$, it would also be chosen in an economy with more information $\left(i^{+}\right)$. This result is not generally true as shown in the following Proposition.

Proposition 2: (i) Assume $a=0$ is an irreversible position. Then if the flexible choice is preferred in a given economy, it is also preferred in an economy with a better information structure: $a^{i^{-}}=1 \Rightarrow a^{i^{+}}=1$. (ii) If $a=0$ is not an irreversible position it may happen that $a^{i^{-}}=1 \Rightarrow a^{i^{+}}=0$.

Proof. (i) When the first period choice is $a=0$, because it is an irreversible position, the optimal intermediate decision will be to choose $b=a$. In this case the expected utility will be the same in the two economies: $U^{i^{-}}(a=0)=U^{i^{+}}(a=0)$.

When the first period choice is $a=1$, thanks to the Marschak and Miyasawa [1968] result, that all expected utility maximizers are at least as well off observing $\tilde{y}$ rather than $\tilde{y}^{\prime}$, we have: $U^{i^{+}}(a=1)=u(1)+E_{\tilde{y}} \max _{c \in\{0,1\}} E_{\tilde{x} / \tilde{y}} v(c \tilde{x}) \geq U^{i^{-}}(a=1)=u(1)+$ $E_{\tilde{y}^{\prime}} \max _{c \in\{0,1\}} E_{\tilde{x} / \tilde{y}^{\prime}} v(c \tilde{x})$. 
So if the flexible choice is optimal in the economy with less information, i.e., $U^{i^{-}}(a=$ 1) $>U^{i^{-}}(a=0)$, then combining the previous inequalities we get: $U^{i^{+}}(a=1) \geq$ $U^{i^{-}}(a=1)>U^{i^{-}}(a=0)=U^{i^{+}}(a=0)$ which concludes the proof.

(ii) We construct an example with two signals and two states where $a^{i^{-}}=1 \Rightarrow a^{i^{+}}=0$. Normalize cost and utility functions so that $C(1)=C>0, u(0)=3 C / 5, u(1)=0$ (the flexible choice gives less utility today) and $v(0)=0$. A first information structure has posterior probabilities $\tilde{x} / y_{1}^{i-}={ }_{d}(7 / 12,5 / 12)$ and $\tilde{x} / y_{2}^{i-}={ }_{d}(1 / 4,3 / 4)$, with signal probabilities $y^{i-}={ }_{d}(3 / 4,1 / 4)$. A second information structure has posterior probabilities $\tilde{x} / y_{1}^{i+}={ }_{d}(3 / 4,1 / 4)$ and $\tilde{x} / y_{2}^{i+}={ }_{d}(1 / 4,3 / 4)$, with signal probabilities $y^{i+}={ }_{d}(1 / 2,1 / 2)$. The prior distribution of lottery $\tilde{x}$ is then $(1 / 2,1 / 2)$.

Assume that the two states are such that $E_{\tilde{x} / y_{1}^{i}} v(\tilde{x})>C$ for $i=i^{+}, i^{-}$and $E_{\tilde{x} / y_{2}^{i}} v(\tilde{x})<$ $0<C$ for $i=i^{+}, i^{-}$. Then, the flexible choice is optimal in the economy with less information, i.e., $U^{i^{-}}(a=1)>U^{i^{-}}(a=0)$, and the rigid one is optimal in the economy with more information, i.e., $U^{i^{+}}(a=0)>U^{i^{+}}(a=1)$. In fact, the two previous inequalities can be rewritten as: $3 C / 4>3 C / 5>C / 2$.

The intuition of the above result is the following. In the first part of the Proposition $a=0$ is an irreversible position. This means that choosing $a=0$ leaves no choice to be made in $t=2$ and information is useless, $U^{i^{-}}(a=0)=U^{i^{+}}(a=0)$. Then, the cost of constraining the second period choice is somehow higher when time 2's decision could have made use of more information. So if the flexible choice $a=1$ is optimal when one expects a given information $\left(i^{-}\right)$, it must also be optimal when one expects more information $\left(i^{+}\right)$. We get the same result as Epstein [1980]. On the contrary, if $a=0$ is not an irreversible position, information has a value, $U^{i^{-}}(a=0) \neq U^{i^{+}}(a=0)$, and the decision maker could well decide to choose the rigid position today and to pay the cost of reversal tomorrow if it turns out to be optimal.

\subsection{Posterior-preserving information improvements}

Another direction of research, to sign the effect of information on the optimal degree of flexibility is to restrict the set of information structures. We simplify our problem by looking at a special kind of information improvement due to Green and Stokey [1980]. The optimal decisions $c\left(a, b, \pi_{y}\right)$ and $b\left(a, \pi_{y}\right)$ depend in general on the posterior distributions implied by the information structure, and these are in general different as the information structure changes. This motivates the following restriction on the set of better information structure. We call a posterior-preserving improvement in information (PPII): an improvement in information structure from $\tilde{y}$ to $\tilde{y}^{\prime}$ satisfying condition (3.3) and such that $\pi_{y}\left(x_{i}\right)=\pi_{y^{\prime}}\left(x_{i}\right) \forall i$ and $y$, i.e. for any realization of the random variable $\tilde{x}$ and for any realization of the signal $\tilde{y}$. Hence for PPII $c\left(a, b, \pi_{y}\right)=c^{*}$ and $b\left(a, \pi_{y}\right)=b^{*}$, decisions are unaffected by the change in information structure. ${ }^{4}$ Exploiting the fact that the only thing that changes is the density of the signals we find the following result:

Proposition 3: A PPII decreases the degree of flexibility. 
Proof. Using definition (3.4) of better information structure, together with the definitions of intermediate and second period value function (3.2) and (3.1) and the derivative of $J\left(a, \pi_{y}\right)(3.6)$ we get

$$
\begin{aligned}
E_{\tilde{y}} J\left(a, \pi_{y}\right) \geq E_{\tilde{y}} J\left(a, \pi_{y^{\prime}}\right) & \equiv E_{\tilde{y}}\left[E_{\tilde{x} / \tilde{y}} v\left(c\left(a, b, \pi_{y}\right) \tilde{x}\right)-J_{a}\left(a, \pi_{y}\right)\right] \\
& \geq E_{\tilde{y}^{\prime}}\left[E_{\tilde{x} / \tilde{y}^{\prime}} v\left(c\left(a, b, \pi_{y^{\prime}}\right) \tilde{x}\right)-J_{a}\left(a, \pi_{y^{\prime}}\right)\right] .
\end{aligned}
$$

Remembering that the posterior decisions are not affected by the change in information structure we note that $E_{\tilde{y}} E_{\tilde{x} / \tilde{y}} v\left(c\left(a, b, \pi_{y}\right) \tilde{x}\right)=E_{\tilde{y}^{\prime}} E_{\tilde{x} / \tilde{y}^{\prime}} v\left(c\left(a, b, \pi_{y^{\prime}}\right) \tilde{x}\right)=E_{\tilde{x}} v\left(c^{*} \tilde{x}\right) \mathrm{im}-$ plying that $E_{\tilde{y}} J_{a}\left(a, \pi_{y}\right) \leq E_{\tilde{y}^{\prime}} J_{a}\left(a, \pi_{y^{\prime}}\right)$. Then, concavity with respect to $a$ of (3.5) gives the result.

A potential intuition for this result is as follows. In general a better information has two effects. First, an irreversibility effect: acquiring information in the future will induce the decision maker to bias his ex ante decision in favour of more flexibility, to make better use of that information. Second, a wealth effect: the implicit increase in wealth generated by better information tends to induce the decision maker to worry less about the future. It increases the willingness to consume in period one, and because flexibility is costly, bias ex ante decision in favour of less flexibility. With a better information structure that is a PPII identical posteriors imply no change in second period choices, therefore the first effect is null and the result follows. ${ }^{5}$

\subsection{Constant relative risk aversion}

In general a better information structure which is not a PPII implies some change in one or both of the best conditional actions. Therefore, the irreversibility effect is active and could dominate the wealth effect. To explore this possibility another direction of research is to study our problem when the utility function belongs to the well-known class of CRRA functions such that:

$$
v(w) \begin{cases}=\frac{w^{1-\alpha}}{1-\alpha} & \text { if } \alpha \neq 1 \\ =\log w & \text { if } \alpha=1\end{cases}
$$

and RRA $=\alpha>0$. To get an explicit solution we also assume that

$$
C(a, b) \begin{cases}=b^{\vartheta} & \text { if } b>a \\ =0 & \text { if } b \leq a\end{cases}
$$

with $\vartheta>1$ to make the cost function increasing and convex.

First, solve the second period constrained maximization problem. The solution to this problem will be a function of previous choices $a$ and $b$, and of information structure: $c\left(a, b, \pi_{y}\right)$. The Kuhn-Tucker expression for $\alpha \neq 1$ is written as ${ }^{6}: K_{c}=\sum_{i=1}^{n} \pi_{y}\left(x_{i}\right) \frac{\left(c x_{i}\right)^{1-\alpha}}{1-\alpha}$ 
$+\lambda(b-c)$, and the Kuhn-Tucker conditions are:

$$
\sum_{i=1}^{n} \pi_{y}\left(x_{i}\right)\left(c x_{i}\right)^{-\alpha} x_{i} \begin{cases}=0 & \text { if } c^{*}<b \\ =\lambda & \text { if } c^{*}=b .\end{cases}
$$

Second, solve the intermediate decision problem. The solution to this problem will be a function of previous choice $a$ and of information structure: $b\left(a, \pi_{y}\right)$. The Kuhn-Tucker expression is written as: $K_{b}=\sum_{i=1}^{n} \pi_{y}\left(x_{i}\right)^{\left(c x_{i}\right)^{1-\alpha}}+\lambda(b-c)-b^{\vartheta} 1_{b>a}$, and the KuhnTucker conditions are:

$$
\vartheta b^{\vartheta-1} 1_{b>a}\left\{\begin{array}{llll}
=0 & \text { if } b^{*} \leq a & \text { and } & c^{*}<b^{*} \\
=\sum_{i=1}^{n} \pi_{y}\left(x_{i}\right)\left(b x_{i}\right)^{-\alpha} x_{i} & \text { if } b^{*}>a & \text { and } & c^{*}=b^{*} .
\end{array}\right.
$$

If after observing the signal the decision maker decides not to pay the cost of reversal, $b^{*} \leq a$, it means that the new information signals that the optimal final decision is to choose a level of $c$ in the interior of the opportunity set, $c^{*}<b^{*} \leq a$. On the contrary, if after observing the signal the decision maker bears the cost to enlarge the second period opportunity set, he will then choose the highest possible level in the set, $c^{*}=b^{*}$.

In this second case, straightforward computations yield $b^{*}=\left(\frac{\vartheta}{\sum_{i=1}^{n} \pi_{y}\left(x_{i}\right) x_{i}^{1-\alpha}}\right)^{\frac{i}{1-\alpha-\vartheta}}$. Then, substituting in (3.6) we get:

$$
J_{a}\left(a, \pi_{y}\right)=\max \left\{0,\left(\frac{\vartheta}{\sum_{i=1}^{n} \pi_{y}\left(x_{i}\right) x_{i}^{1-\alpha}}\right)^{\frac{\vartheta}{1-\alpha-\vartheta}}\right\} .
$$

$J_{a}\left(a, \pi_{y}\right)$ is thus convex in the vector of probabilities when $\alpha<1$. Making use of the Lemma we can state the following Proposition:

Proposition 4: $\quad$ Suppose CRRA. Then a better information structure increases the degree of flexibility if relative risk aversion is smaller than one.

Therefore, when $\alpha<1, J_{a}\left(a, \pi_{y}\right)$ is convex in $\pi_{y}$ and the irreversibility effect holds. Thus, to determine whether a better information structure increases the degree of flexibility, one should check if relative risk aversion is less than unity. Gollier [2001 p. 31] writes that: "it is reasonable to believe that relative risk aversion is somewhere between 1 and 4." If this is true, then the irreversibility effect could not hold.

\section{Conclusion}

For standard irreversibility theory the prospect of acquiring better information in the future should induce less irreversible decisions: the "irreversibility effect". This result relies on the definition of an irreversible position as one that would be technically or economically impossible to reverse. In practice, many positions can be reversed at an affordable cost. In this case, the irreversibility effect should not be given for granted. In fact, if the first period 
choice is restricted to be 'all or nothing' and if the first period choice is technically or economically irreversible then better information promotes flexibility, as in Epstein [1980]. But a lower cost of reversal can make the rigid position preferable. Moreover, if we look at the special information improvements that live posterior distributions unchanged, we find that the value of remaining flexible tends to decrease with better information. Finally, if relative risk aversion is constant (CRRA) and lower than 1, the irreversibility effect holds even when a position is not economically or technically irreversible. But reasonable values for relative risk aversion are somewhere between 1 and 4 .

\section{Acknowledgments}

I thank G. Albano, L. Eeckhoudt, M. Li Calzi, A. Lizzeri and S. Modica for their helpful comments. My greatest debt is to C. Gollier and G. Cestone for their numerous and useful suggestions. A previous version of this paper has been presented at F.U.R. IX.

\section{Notes}

1. The separation-and-transmutation technique consists in separating long living particles from short living ones and in transmuting the latter into short living via a complex process, thus making a lengthy storage of wastes unnecessary.

2. An economically irreversible position implies a cost of reversal higher than the most that can conceivably be gained tomorrow by choosing in an unconstrained set. Of course there is no distinction for an optimizer between a technically irreversible and an economically irreversible position.

3. For the nuclear wastes example the information may concern a possible leakage of radioactivity in the environment, the discovery of a new treatment to reduce the radioactivity of wastes, and so on.

4. A PPII is (with some technical restrictions) a mean preserving spread in the distribution of the signals (Singh [1991]). It should also be noted that if there is a finite number of signals, this must be greater than the number of states (Green and Stokey [1980]).

5. Note that better information leads to higher expected utility even when the posterior actions are unchanged. The reason being that, signals are more conclusive, that is, even when the actions are unchanged there is a smaller risk of choosing the wrong action.

6. For $\alpha=1, c$ and $b$ are independent of $\pi$.

\section{References}

ARROW, K.J. and FISHER, A.C. [1974]: "Environmental Preservation Uncertainty, and Irreversibility,” Quarterly Journal of Economics, 88, 312-319.

BLACKWELL, D. [1951]: “Comparison of Experiments," in University of California Press, Berkeley, J. Neyman (Ed.), Proceedings of the Second Berkeley Symposium on Mathematical Statistics and Probability, 93-102.

EPSTEIN, L.S. [1980]: "Decision-Making and the Temporal Resolution of Uncertainty," International Economic Review, 21, 269-284.

FREIXAS, X. and LAFFONT, J.J. [1984]: “On the Irreversibility Effect,” in Bayesian Models in Economic Theory, M. Boyer and R. Kihlstrom (Eds.), Ch. 7, Elsevier, Dordrecht.

GOLLIER, C. [2001]: The Economics of Risk and Time. Cambridge: The MIT Press.

GOLLIER, C., JULLIEN, B., and TREICH, N. [2000]: "Scientific Progress and Irreversibility: An Economic Interpretation of the "Precautionary Principle," Journal of Public Economics, 75, 229-253.

GREEN, J. and STOKEY, N. [1980]: A Two-Person Game of Information Transmission. H.I.E.R. 751, Harvard University. 
HENRY, C. [1974]: “Investment Decisions Under Uncertainty: The Irreversibility Effect,” American Economic Review, 64, 1006-1012.

JONES, R.A. and OSTROY, J.M. [1984]: "Flexibility and Uncertainty," Review of Economic Studies, LI, 13-32. MARSCHAK, J. and MIYASAWA, K. [1968]: "Economic Comparability of Information Systems," International Economic Review, 137-174.

SINGH, N. [1991]: "Posterior-Preserving Information Improvements and Principal-Agent Relationships," Journal of Economic Theory, 55, 192-202. 